\title{
CAN THE SPOTLIGHT OF ATTENTION BE SHAPED LIKE A DOUGHNUT? Evidence From Steady-State Visual Evoked Potentials
}

\author{
Matthias M. Müller ${ }^{1}$ and Ronald Hübner ${ }^{2}$ \\ ${ }^{1}$ University of Liverpool, Liverpool, England, and ${ }^{2}$ University of Konstanz, Konstanz, Germany
}

\begin{abstract}
Visual attention enables observers to extract and process high-priority information in the visual field. Controversy remains as to whether or not observers can ignore information that falls within the spatial beam of attention. We used an objective physiological measure, the steady-state visual evoked potential (SSVEP), to investigate this question. A stream of flickering small, uppercase letters was embedded in the center of a stream of large, uppercase letters. A unitary beam would result in no difference of the SSVEP amplitude elicited by the small letter stream when it was attended versus ignored (i.e., when subjects attended the large letter stream). Contrary to this prediction, SSVEP amplitude increased by almost $100 \%$ when the small letter stream was attended compared with when it was ignored. The results support the notion that the attentional spotlight can be formed like a doughnut, processing central information differentially depending on whether it is attended or ignored.
\end{abstract}

At any given moment, the brain is confronted with an enormous amount of visual input. To guarantee coherent behavior, selective attention must focus the limited processing resources on the relevant part of the available information while ignoring the rest (e.g., Hillyard, Mangun, Woldorff, \& Luck, 1995; Kinchla, 1992). However, up to the present, it has been an open question exactly how and at which stage of processing this selection takes place. A number of behavioral and electrophysiological studies support the idea that attention modulates the processing of visual input at an early location-based stage (B.A. Eriksen \& Eriksen, 1974; Hillyard, Vogel, \& Luck, 1998; LaBerge, 1995; Mangun, 1995; I.P. Posner \& Petersen, 1990; M.I. Posner \& Dehaene, 1994). This type of selection has been described by the "spotlight" (e.g., I.P. Posner \& Petersen, 1990) or "zoom lens" (C.W. Eriksen \& St. James, 1986) metaphor, which implies that all stimuli that fall into the beam of the spotlight or zoom lens will be processed preferentially. This idea has been supported by studies in which early visual evoked potentials (VEPs) were enhanced for stimuli inside compared with outside the beam of the spotlight (Gomez Gonzales, Clark, Fan, Luck, \& Hillyard, 1994; Hillyard et al., 1998; Luck \& Ford, 1998; Mangun, 1995).

However, results from experiments investigating the selection of superimposed objects have led to an alternative approach that assumes the visual system processes information largely in parallel across the

Address correspondence to Matthias M. Müller, Cognitive Neuroscience and Neuropsychology, Department of Psychology, University of Liverpool, Eleanor Rathbone Building, Liverpool L69 7ZA, United Kingdom, e-mail: m.mueller@liverpool.ac.uk, or to Ronald Hübner, Department of Psychology, University of Konstanz, D-78457 Konstanz, Germany, e-mail: ronald.huebner@ uni-konstanz.de. visual field up to a later stage during which objects are represented, and that attentional selection is based on these representations rather than on location (e.g., Baylis \& Driver, 1993; Duncan, 1984; Hübner \& Backer, 1999; Tipper \& Weaver, 1998). A recent functional magnetic resonance imaging (fMRI) study by O'Craven, Downing, and Kanwisher (1999) supports this view. Their subjects had to attend to either a face or a house, which were transparently superimposed; one of the objects was in motion. O'Craven et al. found differential activation of specific cortical areas depending on which object was attended, a result that was seen as consistent with object-based selection. However, Vecera and Farah (1994) have challenged the object-based approach by suggesting that the selection of superimposed objects can still be explained by location-based mechanisms if one assumes that spatial attention can be focused exactly on the grouped spatial arrays occupied by an object, irrespective of its form.

The grouped-array hypothesis is similar to the assumption that visual attention can be distributed over different discrete areas in the visual field (Castiello \& Umilta, 1992; Kramer \& Hahn, 1995; LaBerge, 1995; Shaw, 1978; Shaw \& Shaw, 1977). Empirical studies investigating this notion have had inconclusive results (cf. Cave \& Bichot, 1999). For instance, when Eimer $(1999,2000)$ had his subjects attend to two noncontiguous ring-shaped regions, he found a reduced VEP negativity for to-be-ignored targets in the region between the to-beattended regions. However, analyses of VEPs to intrusive probe stimuli have led other researchers to claim that attention cannot be focused on different areas simultaneously (Heinze et al., 1994).

In the present study, we investigated attentional selection by superimposing two streams of capital letters presented at different rates. Such repetitive stimulus onsets elicit continuous, nearly sinusoidal brain responses that can be recorded from the human scalp, so-called steady-state visual evoked potentials (SSVEPs; Regan, 1989). It is known that SSVEP amplitude is greater for attended stimuli than for unattended ones (Morgan, Hansen, \& Hillyard, 1996; Müller, Picton, et al., 1998). Moreover, in contrast to the standard VEP method, the SSVEP technique has the advantage that it requires no long interstimulus intervals (ISIs) or intrusive probes, which might attract attention involuntarily.

The crucial point for the present objective is that the letter streams differed not only in presentation rate but also in size. A sample stimulus is shown in Figure 1. Obviously, a spotlight of attention with variable diameter can easily be focused on the small letter without covering the large one, but not vice versa. Therefore, we hypothesized that if selection is location based and attention is distributed in a spotlight manner across the region occupied by the respective letter stream, the SSVEP amplitude for the small letter stream should be the same irrespective of whether subjects attend to the small or to the large letter stream. In addition, in the event-related potential (ERP) of the unattended small letter stream, one could expect a measurable late pos- 


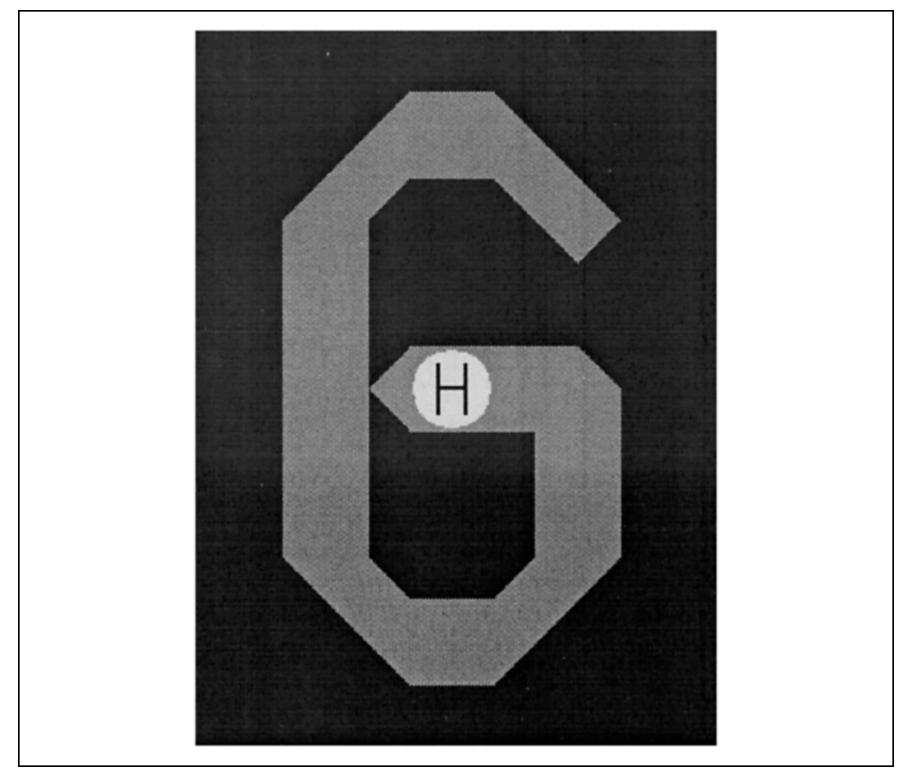

Fig. 1. Schematic representation of a stimulus presented in the experiment.

itive complex (LPC), or P300, ${ }^{1}$ in response to the target's appearance in that stream, even if a large spotlight in this case led to reduced density of attentional resources. If these predictions were not confirmed, then we would conclude that information in the center of the beam is processed differentially depending on whether it is attended or ignored.

\section{METHOD}

\section{Subjects}

Twelve right-handed students (10 males) between the ages of 22 and 33 years $(M=25.4$ years) participated in the study. All had normal or corrected-to-normal vision. Informed consent was obtained from each subject after the nature of the study was fully explained.

\section{Stimuli and Procedure}

The stimuli were large gray capital letters and small capital letters placed in a gray oval in the center of the large letters (see Fig. 1). They were presented on the center of a 21-in. computer monitor situated 1 $\mathrm{m}$ in front of the subjects. The large letters subtended a viewing angle of $11.3^{\circ} \times 5.1^{\circ}$, the central oval subtended a viewing angle of $1.15^{\circ} \times$ $1.72^{\circ}$, and the small uppercase letters were slightly smaller to fit into the oval. The large and small letters were presented in a random stream of letters selected from $A$ to $O$ of the alphabet. Stimulation frequencies were synchronized to the $70-\mathrm{Hz}$ refresh rate of the monitor, resulting in each letter being presented for 10 frames $(=7.0 \mathrm{~Hz})$ in the slow-frequency condition and 6 frames $(=11.67 \mathrm{~Hz})$ in the fast-frequency condition. The central oval also flickered, synchronized to the frequency of the small uppercase letters.

1. The P300 is a positive component in the evoked potential with a latency of 300 to $600 \mathrm{~ms}$. It is found when subjects are required to detect targets in a train of standard stimuli (Picton, 1992), as was the case in the present experiment.
Subjects were instructed to fixate on the center of the screen (the oval) and to either attend to the large letters while ignoring the letters in the oval or vice versa. The stimuli streams were presented for a period of 2,600 ms at either $7.0 \mathrm{~Hz}$ for the large letters and $11.67 \mathrm{~Hz}$ for the oval and small letters or vice versa. The onset for each trial was synchronized. Three main factors were varied: attention (attend vs. ignore), letter size (large vs. small), and frequency (fast vs. slow). Subjects had to detect the target letter $H$ in the to-be-attended stream. This letter was present in $50 \%$ of the trials in both streams and appeared between 500 and 2,000 ms after flicker onset. After each trial, subjects pushed one button if the target had been present in the relevant stream and another button if it had been absent. Each experimental condition was presented in two 80-trial blocks in a randomized block design. Prior to the experiment, subjects performed practice trials to train them to detect targets and to avoid eye movements and blinks during stimulus presentation.

\section{Electroencephalogram (EEG) Recordings and Analysis}

EEG was recorded from electrodes at $\mathrm{Fz}, \mathrm{Cz}, \mathrm{Pz}, \mathrm{Oz}, \mathrm{T} 5, \mathrm{P} 3, \mathrm{O} 1$, T6, P4, O2, and the right mastoid (international 10-20 system), referenced to the left mastoid. In addition, the horizontal and vertical electro-oculogram (EOG) was recorded. The sampling rate was $250 \mathrm{~Hz}$ and preprocessed on-line by a band-pass filter of DC to $100 \mathrm{~Hz}$. Data were stored on disk for off-line analysis.

For EEG analysis, two epochs were extracted. The SSVEP amplitude was analyzed from flicker onset to flicker offset (i.e., 2,600 ms). The P300 was analyzed from $200 \mathrm{~ms}$ before to 1,000 ms after target onset. Each epoch containing electromyogram artifacts or blinks exceeding $75 \mu \mathrm{V}$ was excluded from further analysis. The data from 1 subject were excluded because of excessive artifacts, and the data from an additional subject were excluded because of a very high error rate in two of the conditions. For the remaining 10 subjects, the mean rejection rate was $25.7 \%$ for the SSVEP epochs and $15.8 \%$ for the P300 epochs. Artifact-free epochs were averaged and algebraically rereferenced to averaged mastoids by subtracting one half of the averaged signal recorded from the right mastoid from the averaged signal at each scalp site.

SSVEP amplitude was extracted by means of complex demodulation (Müller, 1998; Müller et al., 1994; Regan, 1989; Rockstroh et al., 1996). The analysis used center frequencies of 11.67 and $7.0 \mathrm{~Hz}$, respectively, and a low-pass filter of $2 \mathrm{~Hz}$, to avoid cross talk between the two frequencies. The amplitudes extracted by complex demodulation correspond to the peak-to-baseline amplitude of the SSVEP. In order to not include the visual evoked response to flicker onset in the SSVEP amplitude, we excluded the first $500 \mathrm{~ms}$ of each trial from further analysis. Thus, mean SSVEP amplitude was calculated for the time window between 500 and 2,500 ms after flicker onset. The P300 was extracted as the mean amplitude in the time window from 450 to $550 \mathrm{~ms}$ after target onset. Mean baseline amplitude was calculated for $200 \mathrm{~ms}$ before target onset and subtracted from the mean P300 amplitude.

For statistical analysis of the SSVEP amplitude, electrodes were clustered to form three regions, left posterior (LP: T5, P3, O1), central (C: $\mathrm{Cz}, \mathrm{Pz}, \mathrm{Oz}$ ), and right posterior (RP: T6, P4, O2). For the P300 analysis, electrode sites $\mathrm{P} 3, \mathrm{Pz}$, and $\mathrm{P} 4$ were chosen.

\section{Statistical Analysis}

SSVEP amplitude was subject to a repeated measures analysis of variance (ANOVA) with factors of attention (attend vs. ignore), fre- 
quency (fast vs. slow), condition (large vs. small), electrode location (LP vs. C vs. RP), and electrode (three electrodes in each cluster). P300 was subject to a repeated measures ANOVA with factors of attention (attend vs. ignore), frequency (fast vs. slow), condition (large vs. small), and electrode (P3 vs. Pz vs. P4). Error rates were subject to a repeated measures ANOVA with factors of target (present vs. absent), frequency (fast vs. slow), and size (large vs. small). For electrophysiological and behavioral data, $p$ values were adjusted by the Huynh-Feldt-epsilon procedure when necessary.

\section{RESULTS}

\section{Behavioral Data}

Table 1 displays the average percentages of false alarms and misses across the 10 subjects for the to-be-attended letter stream. Overall, more errors occurred when the frequency was fast than when it was slow, $F(1,9)=240, p<.001$, and when the attended letters were large rather than small, $F(1,9)=14.92, p<.01$. However, these two factors interacted significantly, $F(1,9)=14.62, p<.01$. The performance difference between large and small letters was more pronounced for the faster presentation rate than for the slow rate (Table 1). Furthermore, misses occurred more often than false alarms, $F(1,9)=$ $25.12, p<.001$. There was a significant interaction with frequency, $F(1,9)=17.73, p<.01$, showing that the difference between misses and false alarms was larger for letters presented at the fast rate compared with the slow rate (Table 1).

\section{Electrophysiological Data}

\section{SSVEPS}

The SSVEPs from an individual subject are shown in Figures 2a and $2 \mathrm{~b}$. These waveforms are moving-window averages (Morgan et al., 1996; Müller, Picton, et al., 1998) over a duration of $428.58 \mathrm{~ms}$ and thus include three cycles of the 7-Hz SSVEP and five cycles of the 11.67-Hz SSVEP. Because of large interindividual differences in the phase of the SSVEP at different scalp sites, the grand average waveforms were not informative and are not shown.

Table 1. Average percentage of false alarms and misses

\begin{tabular}{lcc}
\hline \hline & \multicolumn{2}{c}{ Kind of error } \\
\cline { 2 - 3 } Frequency and size & False alarms & Misses \\
\hline $11.67 \mathrm{~Hz}$, large letters & 18.13 & 31.63 \\
& $(8.7)$ & $(3.7)$ \\
$7.0 \mathrm{~Hz}$, large letters & 5.50 & 7.38 \\
& $(1.4)$ & $(1.9)$ \\
$11.67 \mathrm{~Hz}$, small letters & 10.62 & 23.63 \\
& $(1.7)$ & $(2.6)$ \\
$7.0 \mathrm{~Hz}$, small letters & 3.38 & 4.38 \\
& $(1.0)$ & $(1.1)$ \\
\hline
\end{tabular}

Note. Standard errors are in parentheses.

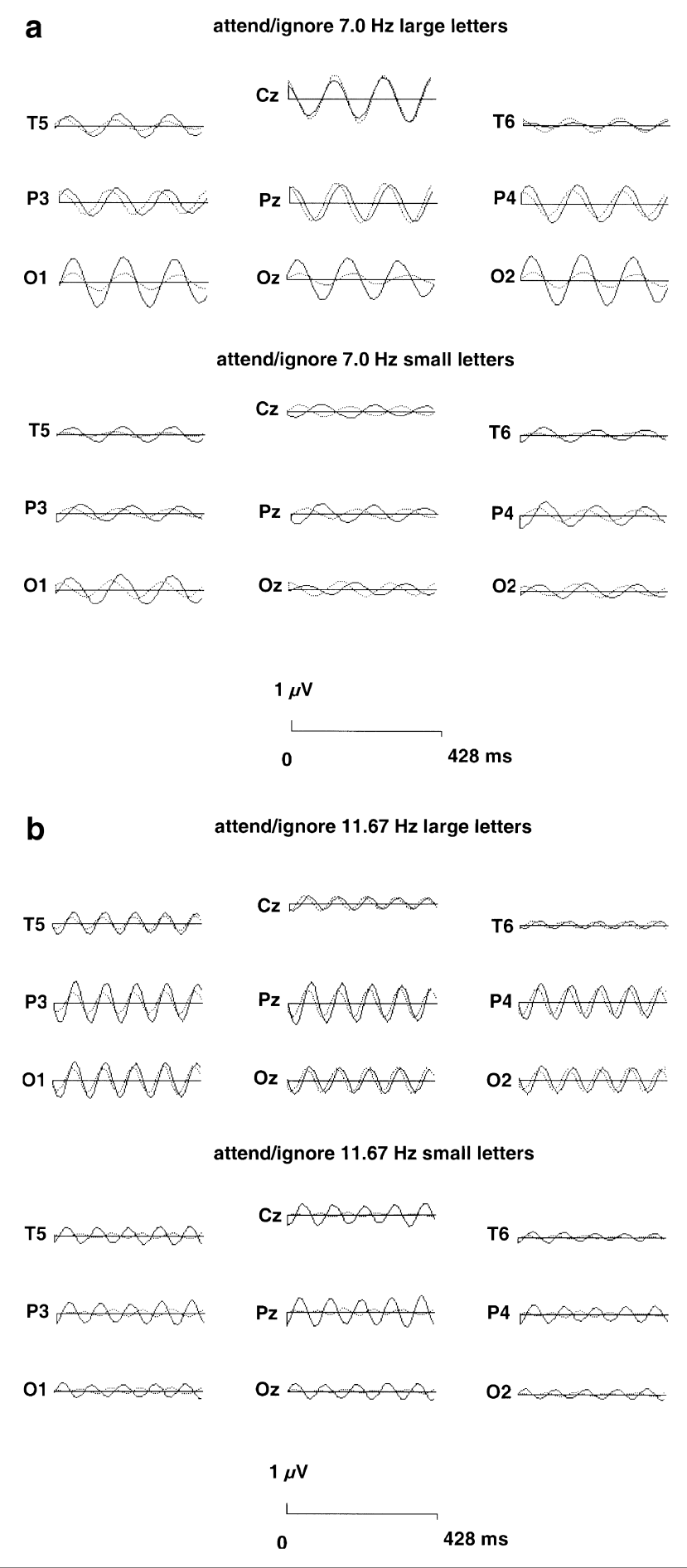

Fig. 2. Steady-state visual evoked potentials (SSVEPs) recorded from posterior electrodes from a representative subject. The waveforms shown were averaged using a moving-window technique. Solid lines represent the SSVEP waveforms for the attended stimulus train; dashed lines represent the waveforms when the same stimulus train was ignored. Results are shown separately for the $7-\mathrm{Hz}$ (a) and $11.67-\mathrm{Hz}$ (b) streams. In each case, the upper panel shows results for the large letter stream, and the lower panel shows results for the small letter stream. 
Overall, the main effect of frequency was similar to the effect demonstrated in previous experiments (Müller \& Hillyard, 2000; Müller, Picton, et al., 1998): The SSVEP for the slow stream was greater than the SSVEP for the fast one, $F(1,9)=9.57, p<.05$. Amplitudes were also larger for the large letter stream compared with the small one, $F(1,9)=6.20, p<.05$. Most important, SSVEP amplitudes were significantly enhanced for the attended letter stream compared with the ignored one, $F(1,9)=18.53, p<.005$. However, there was a reliable interaction between attention and frequency, $F(1,9)=9.00, p<.05$, indicating that the effect of attention on the SSVEP amplitude was larger for the slow stream $(0.70 \pm 0.05 \mu \mathrm{V}$, baseline-to-peak, vs. $0.47 \pm$ $0.04 \mu \mathrm{V})$ than for the fast stream $(0.51 \pm 0.04 \mu \mathrm{V}$ vs. $0.39 \pm 0.03 \mu \mathrm{V})$. There was also a three-way interaction among attention, frequency, and size, $F(1,9)=11.61, p<.01$. As can be seen in Figure 3, and was confirmed by post hoc tests, in the case of the large letter stream, a significant attention effect occurred only for the slow frequency. In the case of the small letter stream, there was no significant difference between the fast and slow frequencies, and attention caused an increase of almost $100 \%$ in the mean SSVEP amplitude $(0.48 \pm 0.03$ $\mu \mathrm{V}$ vs. $0.25 \pm 0.01 \mu \mathrm{V}), F(1,9)=26.70, p<.001$.

As depicted in Figure 3, the largest SSVEP amplitude for each condition was obtained across the central electrodes $(\mathrm{Oz}, \mathrm{Pz}, \mathrm{Cz})$ for both large and small letters; the second-largest amplitude was at the left parietal electrodes. The Attention $\times$ Frequency $\times$ Condition $\times$ Electrode Location interaction was significant, $F(2,18)=5.54, p<.05$.

\section{P300}

Figure 4 depicts the baseline-corrected grand mean P300 amplitudes for targets in the attended and unattended streams for electrodes P3, Pz, and P4. As expected, the P300 was larger for attended targets than for ignored ones, $F(1,9)=70.30, p<.001$. The interaction with frequency, $F(1,9)=28.54, p<.001$, indicated that the difference was larger for the slow frequency than for the fast one. Furthermore, the largest P300 to attended targets was measured at electrode site Pz, $F(2,18)=15.04, p<.001$, for the Attention X Electrode interaction. This difference was also more pronounced for the slow than for the fast frequency, $F(2,18)=14.95, p<.005$, for the Attention $\times$ Frequency $\times$ Electrode interaction. There were no significant effects or interactions involving letter size.

\section{DISCUSSION}

The aim of the present experiment was to investigate the mechanisms of visual attentional selection. Is this selection based on location or on object representations? When it is location based, must attention be distributed over a contiguous beamlike area, or is the distribution more flexible? To answer these questions, we asked our subjects to detect a target in one of two superimposed letter streams that differed in size. The SSVEP amplitude associated with the small stream increased $100 \%$ when this stream was attended compared with when it

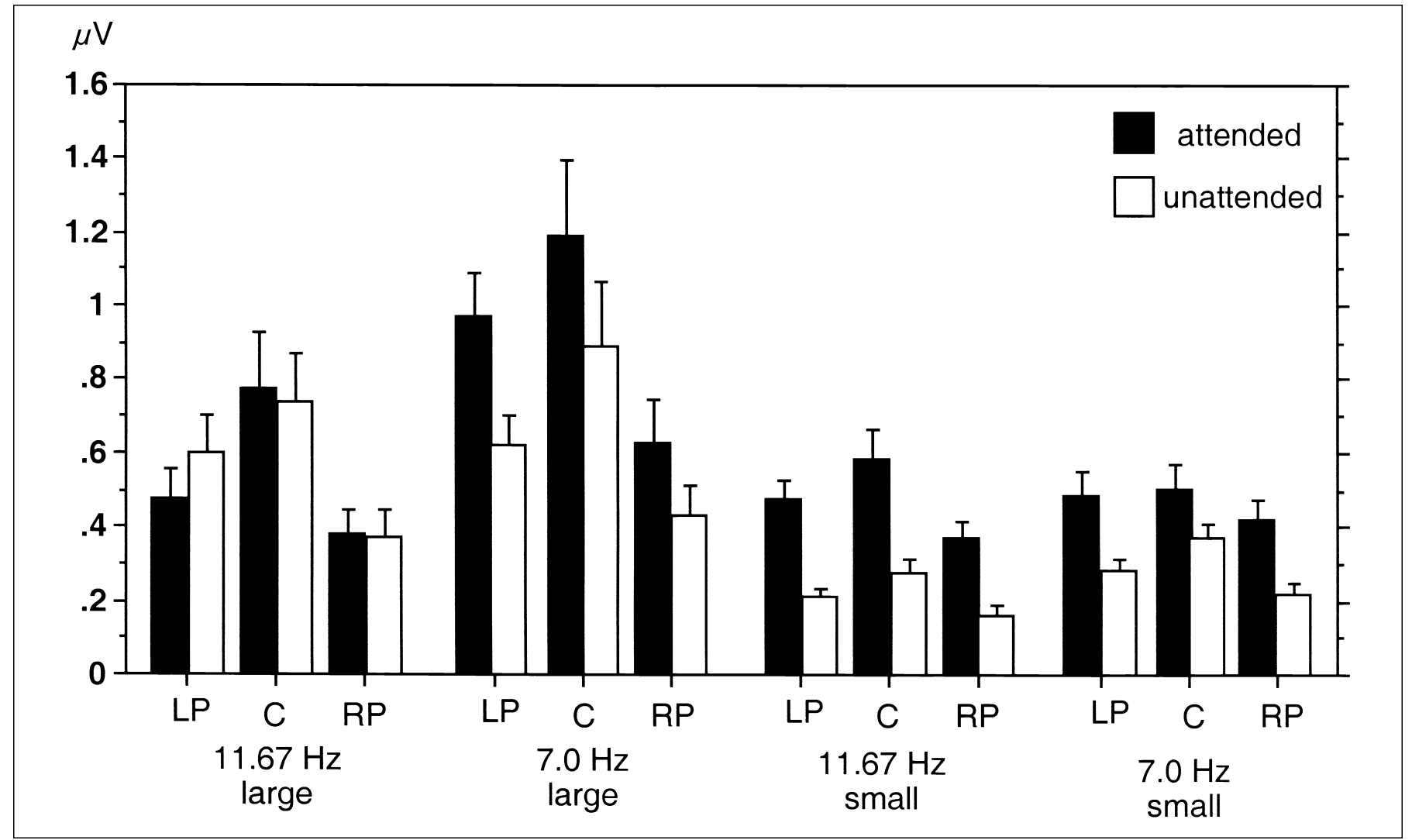

Fig. 3. Mean baseline-to-peak amplitude (plus standard error) of the steady-state visual evoked potential (SSVEP) averaged across 10 subjects, for three location clusters (each comprising three electrodes): LP (left parietal), C (central), and RP (right parietal). Results are shown separately for attended and unattended large and small letters presented at a rate of 11.67 or $7.0 \mathrm{~Hz}$. 


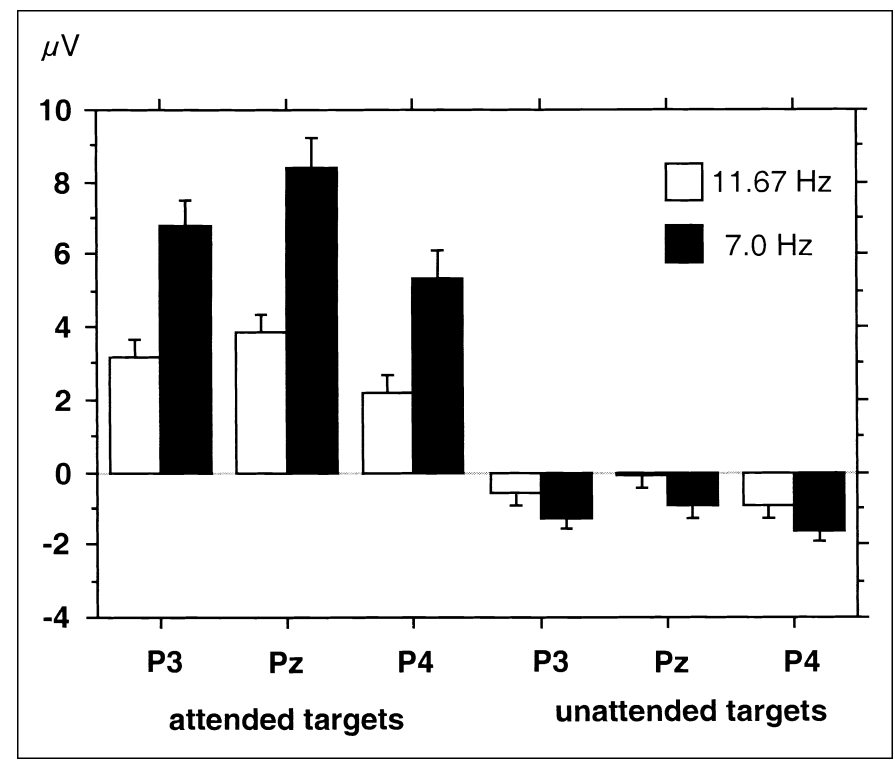

Fig. 4. Mean baseline-corrected $\mathrm{P} 300$ amplitude (plus standard error) averaged across 10 subjects, for electrodes P3, Pz, and P4. Results are shown separately for the attended and unattended targets in the 11.67or $7.0-\mathrm{Hz}$ letter stream.

was not attended (i.e., subjects attended to the large letter stream). Thus, contrary to Heinze et al. (1994), we found clear evidence that the neural responses to stimuli located within the spotlight of attention are significantly different when they are ignored as opposed to attended. With respect to the spotlight metaphor, this can only be explained with an annular-shaped beam.

Furthermore, our data are, to some extent, in line with the results of Eimer $(1999,2000)$. When his subjects attended to two separated ringshaped regions, he found a reduced negativity for to-be-ignored information located between theses regions, a finding that is also at odds with the zoom-lens or spotlight model. However, our results go a step further by showing that even the center of the beam can be ignored when subjects are instructed to maintain focus on centrally presented stimuli.

With respect to the SSVEP, our results confirm previous findings of a bigger amplitude for lower than for higher stimulation frequencies (Müller, 1998; Müller, Picton, et al., 1998; Müller, Teder-Sälejärvi, \& Hillyard, 1998). In the present experiment, we controlled for this by balancing the conditions. In addition, we wanted to control for possible differences in the difficulty of detecting targets in the fast and slow streams. Interestingly, most errors occurred in the $11.67-\mathrm{Hz}$, large-letter condition, which also showed only a small effect of attention on the SSVEP. It seems obvious that the high proportion of errors in this condition was not the consequence of the $7.0-\mathrm{Hz}$ small letter stream having attracted more attention than the $11.67-\mathrm{Hz}$ large letter stream or of attention shifting between the two streams, because we found no P300 for the to-be-ignored targets in the small letter stream (see Fig. 4). Attending to the large letter stream could have required a strong inhibition of the small stream, but a suppression of the small letter stream would have been equally required in the condition in which subjects had to attend to the slow large letter stream (ignoring the fast small stream), and in this case the SSVEP attention effect was very pronounced (see Fig. 3).
Given the attentional modulation of the SSVEP for the small letter stream and the high error rate for the large stream in our experiment, an alternative explanation might be derived from the zoom-lens model (C.W. Eriksen \& St. James, 1986). Often, it is assumed that attentional resources are distributed homogeneously within the beam, implying that the density varies with beam size (Barriopedro \& Botella, 1998). This could account for our SSVEP and error data. However, even if density were reduced, one would expect a measurable target P300 in the ignored small letter stream (Picton, 1992), which was not the case (see Fig. 4). This reasoning holds especially true for a gradient model of attention in which most resources are located in the center of the beam (LaBerge, 1995).

Could our results be explained by assuming that the subjects attended to different spatial frequencies rather than to different spatial areas in the two conditions? The problem with such an account is that, although the large letters contained some low spatial frequencies outside the range of the small letters, the opposite was not the case. That is, the spatial-frequency range of the small letters was also part of the spectrum of the large letters. Therefore, global spatial-frequency filtering would not provide an appropriate selection method. Rather, some kind of spatial selection was necessary as well. A mixture of spatial and spatial-frequency-band selection is exactly the mechanism widely assumed for the human visual system. The spatial-frequency band selected by a visual filter covaries with its spatial extension in the visual field (cf. DeVelois \& DeVelois, 1988).

Taken together, the results indicate that the selection of large or small letters in our experiment proceeded in a rather flexible spacebased manner, such that it was even possible to employ an attentional spotlight with an annular shape. However, a serious alternative explanation that remains to be addressed is the object-based account. It could be that our subjects selected the letters within the relevant stream at a late stage by means of attending to characteristic object features. Although it has been shown that such late object selection is possible (Hübner \& Backer, 1999), it is highly unlikely that this type of selection was applied in the present experiment. Rather, there is some empirical evidence that the observed modulation of the SSVEP amplitude was related to early perceptual processes. A previous combined SSVEP and fMRI study with flicker frequencies of 8.6 and 12 $\mathrm{Hz}$ (very close to the frequencies used in the present study) showed an attention-related enhancement of flicker-evoked activity primarily in ventral (areas 18 and 19) and to a much lesser extent lateral (areas 19 and 37) extrastriate cortex of the occipital lobe (Hillyard et al., 1997). Furthermore, in a magentoencephalography study, we found the location of the current dipoles of the 6- and $11.2-\mathrm{Hz}$ steady-state visual evoked field clearly in the vicinity of the calcarine fissure, with very little interindividual variability (Müller, Teder, \& Hillyard, 1997). This localization of the SSVEP would to some extent exclude a late selection process. However, in a recent imaging study, activation of V1 and extrastriatal areas was reported when subjects were involved in an object-based task (Fink, Dolan, Halligan, Marshall, \& Frith, 1997). Object-based activity was even larger than space-based activity in lefthemisphere areas 17 and 18. However, a closer inspection of the stimuli reveals that it is possible that the subjects performed the objectbased task by using spatial information.

In a recent study, we recorded the SSVEP and VEP to color-change stimuli concurrently and found a strong correlation between the SSVEP and N1, as well as between SSVEP and N2 attention effects (Müller \& Hillyard, 2000). Although the correlation between SSVEP and N2 would support to some extent an object-based approach, because the 
$\mathrm{N} 2$ is linked to processing of the target's features and early target recognition processes (e.g., Anllo-Vento \& Hillyard, 1996; Deacon, Breton, Ritter, \& Vaughan, 1991), the correlation between the SSVEP and N1 suggests the SSVEP is an index of an attentional mechanism that facilitates visual processing within the spotlight of spatial attention (Hillyard et al., 1998). Moreover, according to the object-based approach of Fink et al. (1997), we should have obtained more left-hemisphere than right-hemisphere activity, which was not the case. Presently, there is no convincing empirical evidence that the SSVEP is linked to late selection processes. Rather, there is evidence that the SSVEP is linked to an early sensory gain-control mechanism that enhances the signal-to-noise ratio and hence the discriminability of attended-location stimuli (Hillyard et al., 1998; Müller, Picton, et al., 1998; Müller, Teder-Sälejärvi, \& Hillyard, 1998).

To sum up, although we cannot definitively rule out that subjects performed the task by employing some kind of object-based strategy, it is highly unlikely that they did, because there is empirical evidence that the SSVEP is strongly linked to early location-based neural mechanisms. Also, explanations based on other possible selection strategies, such as attention to spatial frequencies, can be rejected. Thus, the most likely account for our data is that the stimulus selection was accomplished by means of a space-based attentional spotlight or zoom lens. Most important, however, the present experiment has convincingly shown for the first time that stimuli that are within the beam of the spotlight can nevertheless be ignored. In the present case, the beam for selecting the large letters while ignoring the small ones must have been shaped like a doughnut.

Acknowledgments-We would like to thank Christian Altman for programming the stimulus presentation, Peter Malinowski for analyzing the behavioral data, and Heidi Messmer, Eva Bonna, and Ursula Lommen for helping record and analyze the data. Furthermore, we would like to thank Steven Hillyard for valuable comments on a first version of our manuscript. This research was supported by grants from the Deutsche Forschungsgemeinschaft.

\section{REFERENCES}

Anllo-Vento, L., \& Hillyard, S.A. (1996). Selective attention to the color and direction of moving stimuli: Electrophysiological correlates of hierarchical feature selection. Perception \& Psychophysics, 58, 191-206.

Barriopedro, M.I., \& Botella, J. (1998). New evidence for the zoom lens model using the RSVP technique. Perception \& Psychophysics, 60, 1406-1414.

Baylis, G.C., \& Driver, J. (1993). Visual attention and objects: Evidence for hierarchical coding. Journal of Experimental Psychology: Human Perception and Performance, $19,451-470$.

Castiello, U., \& Umilta, C. (1992). Splitting focal attention. Journal of Experimental Psychology: Human Perception and Performance, 18, 837-848.

Cave, K.R., \& Bichot, N.P. (1999). Visuospatial attention: Beyond a spotlight model. Psychonomic Bulletin \& Review, 6, 204-223.

Deacon, D., Breton, F., Ritter, W., \& Vaughan, H.G., Jr. (1991). The relationship between N2 and N400: Scalp distribution, stimulus probability, and task relevance. Psychophysiology, 28, 185-200.

DeVelois, R.L., \& DeVelois, K.K. (1988). Spatial vision. Oxford, England: Oxford University Press.

Duncan, J. (1984). Selective attention and the organization of visual information. Journal of Experimental Psychology: General, 113, 501-517.

Eimer, M. (1999). Attending to quadrants and ring-shaped regions: ERP effects of visual attention in different spatial selection tasks. Psychophysiology, 36, 491-503.

Eimer, M. (2000). An ERP study of sustained spatial attention to stimulus eccentricity. Biological Psychology, 52, 205-220.
Eriksen, B.A., \& Eriksen, C.W. (1974). Effects of noise letters upon the identification of a target letter in a nonsearch task. Perception \& Psychophysics, 16, 143-149.

Eriksen, C.W., \& St. James, J.D. (1986). Visual attention within and around the field of focal attention. Perception \& Psychophysics, 45, 225-240.

Fink, G.R., Dolan, P.W., Halligan, P.W., Marshall, J.C., \& Frith, C.D. (1997). Space-based and object-based visual attention: Shared and specific neural domains. Brain, 120, 2013-2028.

Gomez Gonzales, C.M., Clark, V.P., Fan, S., Luck, S.J., \& Hillyard, S.A. (1994). Sources of attention-sensitive visual event-related potentials. Brain Topography, 7, 41-51.

Heinze, H.-J., Luck, S.J., Münte, T.F., Gös, A., Mangun, G.R., \& Hillyard, S.A. (1994) Attention to adjacent and separate positions in space: An electrophysiological analysis. Perception \& Psychophysics, 56, 42-52.

Hillyard, S.A., Hinrichs, H., Tempelmann, C., Morgan, S.T., Hansen, J.C., Scheich, H., \& Heinze, H.J. (1997). Combining steady-state visual evoked potentials and fMRI to localize brain activity during selective attention. Human Brain Mapping, 5, 287-292.

Hillyard, S.A., Mangun, G.R., Woldorff, M.G., \& Luck, S.J. (1995). Neural systems mediating selective attention. In M.S. Gazzaniga (Ed.), The cognitive neurosciences (pp. 665-681). Cambridge, MA: MIT Press.

Hillyard, S.A., Vogel, E.K., \& Luck, S.J. (1998). Sensory gain control (amplification) as a mechanism of selective attention: Electrophysiological and neuroimaging evidence. Philosophical Transactions of the Royal Society of London B, 353, 1257-1270.

Hübner, R., \& Backer, G. (1999). Perceiving spatially inseparable objects: Evidence for feature-based object selection not mediated by location. Journal of Experimental Psychology: Human Perception and Performance, 25, 1556-1567.

Kinchla, R.A. (1992). Attention. Annual Review of Psychology, 43, 711-742.

Kramer, A.F., \& Hahn, S. (1995). Splitting the beam: Distribution of attention over noncontiguous regions of the visual field. Psychological Science, 6, 381-386.

LaBerge, D. (1995). Attentional processing. Cambridge, MA: Harvard University Press

Luck, S.J., \& Ford, M.A. (1998). On the role of selective attention in visual perception. Proceedings of the National Academy of Sciences, USA, 95, 825-830.

Mangun, G.R. (1995). Neural mechanisms of visual selective attention. Psychophysiology, $32,4-18$.

Morgan, S.T., Hansen, J.C., \& Hillyard, S.A. (1996). Selective attention to stimulus location modulates the steady state visual evoked potential. Proceedings of the National Academy of Sciences, USA, 93, 4770-4774.

Müller, M.M. (1998). Oscillatory cortical activities in the human brain. Habilitation thesis, University of Konstanz, Konstanz, Germany. Available from the Universität Konstanz Web site: http://www.ub.uni-konstanz.de/kops/volltexte/2000/376/

Müller, M M., \& Hillyard, S.A. (2000). Concurrent recording of steady-state and transient event-related potentials as indices of visual spatial selective attention. Clinical Neurophysiology, 111, 1544-1552.

Müller, M.M., Picton, T.W., Valdes-Sosa, P., Riera, P., Teder-Sälejärvi, W., \& Hillyard, S.A. (1998). Effects of spatial selective attention on the steady-state visual evoked potential in the 20-28 Hz range. Cognitive Brain Research, 6, 249-261.

Müller, M M., Rockstroh, B., Berg, P., Wagner, M., Elbert, T., \& Makeig, S. (1994). SSRmodulation during slow cortical potentials. In C. Pantev, T. Elbert, \& B. Lütkenhöner (Eds.), Oscillatory event-related brain dynamics (pp. 325-341). New York: Plenum Press.

Müller, M.M., Teder, W., \& Hillyard, S.A. (1997). Magentoencephalographic recording of steady-state visual evoked cortical activity. Brain Topography, 9, 163-168.

Müller, M.M., Teder-Sälejärvi, W., \& Hillyard, S.A. (1998). The time course of cortical facilitation during cued shifts of spatial attention. Nature Neuroscience, 1, 631-634.

O'Craven, K., Downing, P., \& Kanwisher, N. (1999). fMRI evidence for objects as the units of attentional selection. Nature, 401, 584-587.

Picton, T.W. (1992). The P300 wave of the human event-related potential. Journal of Clinical Neurophysiology, 9, 456-479.

Posner, I.P., \& Petersen, S.E. (1990). The attention system of the human brain. Annual Review of Neuroscience, 13, 25-42.

Posner, M.I., \& Dehaene, S. (1994). Attentional networks. Trends in Cognitive Sciences, $17,75-79$.

Regan, D. (1989). Human brain electrophysiology: Evoked potentials and evoked magnetic fields in science and medicine. New York: Elsevier.

Rockstroh, B., Müller, M.M., Heinz, A., Wagner, M., Berg, P., \& Elbert, T. (1996). Modulation of auditory responses during oddball tasks. Biological Psychology, 43, 41-55.

Shaw, M.L. (1978). A capacity model for reaction time. Journal of Experimental Psychology: Human Perception and Performance, 4, 586-598.

Shaw, M.L., \& Shaw, P. (1977). Optimal allocation of cognitive resources to spatial locations. Journal of Experimental Psychology: Human Perception and Performance, 3 , 201-211.

Tipper, S.P., \& Weaver, B. (1998). The medium of attention: Location based, object centered, or scene based. In R.D. Wright (Ed.), Visual attention (pp. 77-107). New York: Oxford University Press.

Vecera, S.P., \& Farah, M.J. (1994). Does visual attention select objects or locations? Journal of Experimental Psychology: General, 123, 146-160.

(RECEIVED 9/18/00; REVISION ACCEPTED 5/21/01) 